\title{
La equidad de género en la educación intercultural del nivel superior: una mirada hacia el respeto de los derechos humanos de las mujeres indígenas y afrodescendientes
}

\section{Resumen}

El presente artículo aborda el tema de la equidad de género desde una mirada del respeto a los derechos humanos de las personas que pertenecen a la comunidad educativa intercultural del nivel superior. A través de una revisión bibliográfica de diferentes estudios escritos sobre el tema, se toma como referencia la colonialidad del poder desde la interseccionalidad raza/ género de María Lugones con el fin de poder explicar el por qué históricamente se dan las diferentes formas de exclusión, discriminación e inequidad hacia las mujeres indígenas y afrodescendientes.

Este trabajo evidencia la brecha que existe entre el acceso, la permanencia y el egreso en las Instituciones del Nivel Superior de indígenas y afrodescendientes, e igualmente, explica las situaciones de desventaja en cuanto a la pertinencia y estrategias pedagógicas, como también el uso de las Tecnologías de la Información (TICs) en los diferentes espacios educativos en los lugares de origen. También se asienta una mirada crítica ante las desigualdades en los espacios educativos del nivel superior para así exigir la creación de políticas educativas que tengan como prioridad asegurar la profesionalización.

Palabras clave: Género; Educación; Colonialidad; Pueblos Originarios y Afrodescendientes.

POR NOLVIA VERÓNICA LÓPEZ RECINOS. Catedrática de la Universidad Pedagógica Nacional Francisco Morazán, Departamento de Ciencias de la Educación. Estudiante del Programa Latinoamericano del doctorado en Educación, Políticas Públicas y Profesión Docente. nolvial@upnfm.edu.hn 


\section{Abstract}

This article addresses the issue of gender equity from a view of respect for the human rights of people belonging to the intercultural educational community in superior education.

Bibliographic reviews from different written studies on the subject were used as a reference in this material, and it takes the coloniality of power from the intersection of race and gender of Maria Lugones to explain why there have been different forms of exclusion, discrimination, and inequity towards indigenous and Afro-descendant women historically.

Quantitate data shows the gap between access, permanence, and conclusion of indigenous and Afrodescendant people in superior education programs. In the same way, there are explanations regarding the disadvantages in terms of relevance, pedagogical strategies, and the use of Tics in different educational spaces in their places of origin as well. One must have a critical look at inequalities in superior education spaces and thus require the creation of educational policies that ensure professionalization as a priority.

Keywords: Gender; Education; Coloniality; Indigenous and Afro-descendant people.

\section{Resumo}

Este artigo aborda a questão da equidade de gênero a partir de uma visão do respeito aos direitos humanos das pessoas pertencentes à comunidade educacional intercultural ao mais alto nível.

Documentado através de uma revisão bibliográfica de diferentes estudos escritos sobre o tema, tomando como referência a colonialidade do poder, a partir da intercorrentalidade raça/gênero de Maria Lugones, a fim de explicar porque historicamente é dar diferentes formas de exclusão, discriminação e iniqüidade para as mulheres indígenas e afrodescendentes.

Este trabalho demonstra com dados quantitativos a lacuna entre acesso, permanência e egresso nas instituições do nível superior de indígenas e afrodescendentes, e também explica as situações de desvantagem em termos de relevância e estratégias pedagógicas, bem como a utilização de TICs em diferentes espaços educativos nos locais de origem. Além disso, uma visão crítica é dada às desigualdades nos espaços educacionais do nível mais alto e, assim, demanda a criação de políticas educacionais que tenham como prioridade garantir a profissionalização.

Palavras chave: Gênero; Educação; Colonialidade; Povos originais e afrodescendentes.

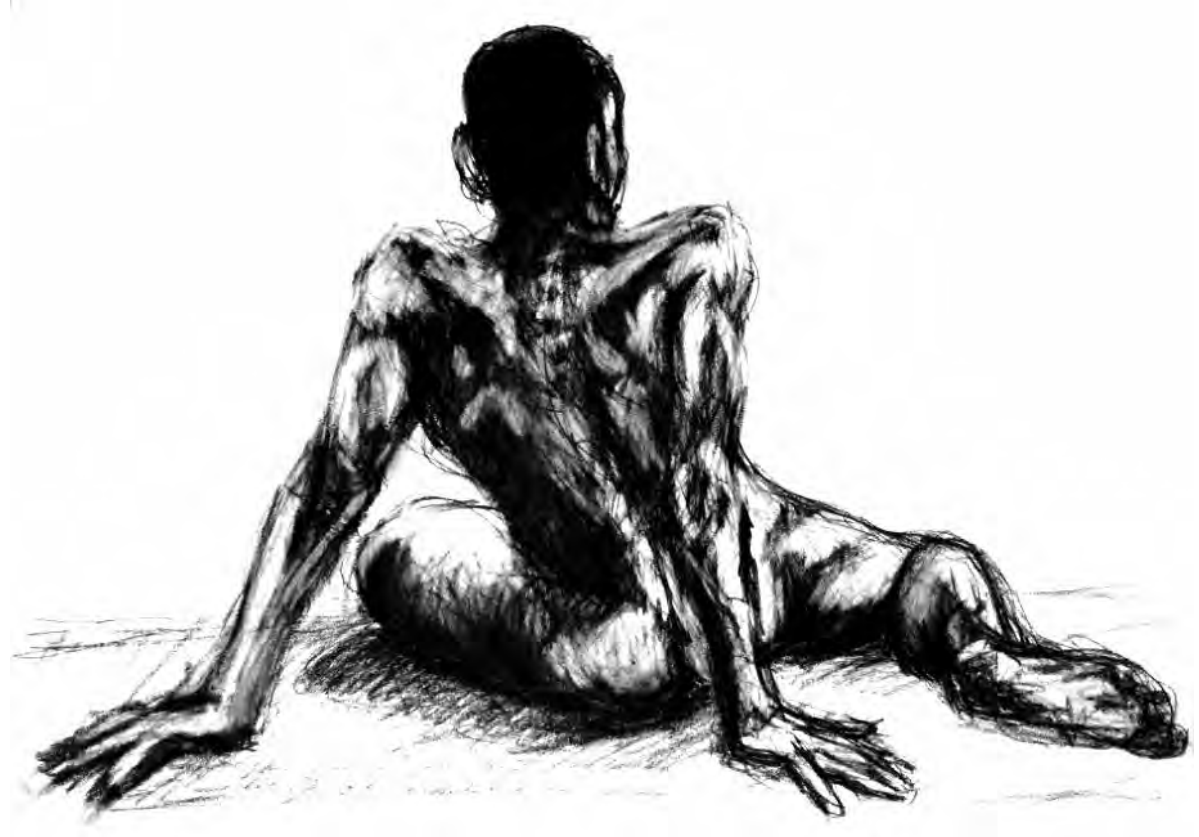




\section{Introducción}

Este artículo se ha escrito con la finalidad de visibilizar las diferentes formas que excluyen y discriminan en los distintos niveles educativos a las personas que pertenecen a poblaciones indígenas y afrodescendientes. Se explica cómo éstas han sido despojados de lo que les pertenece, sobre todo, se abordará el no tener, en la mayoría de los casos, el acceso, ingreso, permanencia y el egreso en instituciones del nivel superior.

Si bien es cierto que se han creado universidades interculturales, también es cierto que persisten problemas de orden académico, ya que se carece de condiciones adecuadas como la pertinencia en las adecuaciones curriculares que, en la mayoría de las veces, no se aplican dentro del aula, por lo que los estudiantes todavía enfrentan situaciones de racismo y otras discriminaciones.

Es por esa razón que se deben visibilizar todas las formas de discriminación con el objetivo de fortalecer programas para facilitar el acceso y permanencia del estudiante en el nivel superior, promover centros regionales donde haya presencia indígena y afrodescendiente y también fomentar programas pertinentes con alto contenido de virtualidad y técnico- tecnológicos.

\section{Equidad de género en las Instituciones del Nivel Superior}

Es posible observar claramente las diferencias de género dentro de los espacios educativos, así como la no aplicación de la educación como derecho humano, ya que éste no sólo debiese considerar el acceso a la educación superior, sino también la permanencia en la misma. Comprender qué sucede en el trayecto que recorren tanto los hombres, como las mujeres dentro de estas áreas es muy importante porque se entenderá cómo es la relación de poder entre los sexos y cómo esa relación se internaliza en la vida de las personas; en el caso que abordaremos, la participación de las mujeres es casi nula en comparación con la de los hombres en actividades donde se pone en juego el quehacer científico alrededor del que se construye un ambiente de violencia simbólica hacía el sexo femenino.

José María Duarte y José Baltazar García (2016) afirman que se debe poner atención a las desigualdades de género relacionadas con la educación, hacen énfasis en el artículo $1^{\circ}$ de la Declaración Universal de los Derechos Humanos (1948) que destaca que todos los seres humanos nacen libres e iguales en dignidad y derechos, y dotados como están de razón y conciencia, deben de comportarse fraternalmente los unos con los otros (p.11). De tal manera que el ideal de la igualdad de género significaría que tanto hombres, como mujeres deberían de disfrutar de los mismos derechos sin ningún tipo de cuestionamiento, asimismo, ser iguales en garantías y oportunidades tanto económicas, como educativas y sociales.

En tal sentido, Mato (2012) reordena datos sobre el promedio de años de estudio de la población adulta indígena comparada con la no indígena, con lo que evidencia la desigualdad de oportunidad de ingreso, permanencia y egreso de las poblaciones originarias, en comparación con la población adulta no indígena, la cual se muestra en el siguiente cuadro:

\section{Promedio años de estudio para población adulta indígena y no indígena de algunos países (mayores de 15 años, ambos sexos)}

\begin{tabular}{|l|c|c|}
\hline \multicolumn{1}{|c|}{ País } & $\begin{array}{c}\text { Promedio de } \\
\text { años de estudio } \\
\text { de población } \\
\text { adulta indígena }\end{array}$ & $\begin{array}{c}\text { Promedio de años } \\
\text { de estudio de } \\
\text { población adulta no } \\
\text { indígena }\end{array}$ \\
\hline Bolivia & 6.5 & 8.8 \\
\hline Brasil & 4.4 & 6.3 \\
\hline Chile & 7.9 & 9.8 \\
\hline Costa Rica & 4.8 & 7.6 \\
\hline Ecuador & 4.1 & 7.8 \\
\hline Guatemala & 2.6 & 5.8 \\
\hline Honduras & 3.7 & 5.3 \\
\hline México & 4.5 & 7.8 \\
\hline Panamá & 4.1 & 8.9 \\
\hline Paraguay & 2.1 & 7.2 \\
\hline Venezuela & 4.7 & 8.6 \\
\hline
\end{tabular}

Fuente: CELADE-CEPAL, 2007. Obtenida en SISPPI (Sistema de Indicadores Sociodemográficos de Poblaciones y Pueblos Indígenas de América Latina). Disponible en: http://www.sisppi.org/redatam/PRYESP/ SISPPI/ [Consultado: 31/07/2007]. 
Es así como la Comisión Interamericana de $\mathrm{Mu}-$ jeres (2002) sostiene que:

...la igualdad de género significa que la mujer y el hombre disfrutan de la misma situación y que tienen iguales condiciones para la plena realización de sus derechos humanos y su potencial de contribuir al desarrollo político, económico, social y cultural, como así también de beneficiarse de los resultados (p. 14).

\section{Colonialidad del poder}

Para entender la relación de género en la educación intercultural hay que abordar la época del colonialismo en América Latina desde la mirada de la colonialidad del poder y la interseccionalidad raza/género para explicar por qué se dan históricamente las diferentes formas de exclusión, discriminación e inequidad hacia las poblaciones indígenas y afrodescendientes.

Según estas feministas africanas e indígenas, no existian en las sociedades yorubas ni en los pueblos indigenas de América del Norte un principio organizador parecido al de género en occidente antes de la colonización. Sus culturas de origen no dividian ni jerarquizaban sus sociedades en base a género, teniendo las mujeres acceso igualitario al poder público y simbólico.

En ese sentido, Aníbal Quijano (2008) acuña el término colonialidad del poder para describir el patrón de poder que se ejerce a lo largo de América con la corona española en el siglo XVI y que luego se extiende a todo el continente de lo que hoy son las personas del tercer mundo: amerindios, africanos, afrodescendientes de El Caribe, América del Sur, Centro y Norte, asiáticos árabes y mestizos (Mendoza, 2010, p.3).

De tal manera, la formación del mundo colonial del capitalismo dio lugar a una estructura de poder, donde una de ellas fue la producción de nuevas iden- tidades históricas, asignadas como "indio", "negro", "blanco" y "mestizo", impuestas después como las categorías básicas de las relaciones de dominación y como fundamento de una cultura de racismo y etnicismo (Quijano, 2014).

El racismo y el etnicismo fueron producidos inicialmente en América y desde hace 500 años no han dejado de ser componentes básicos de estas relaciones de poder. Aunque se haya extinguido el colonialismo como sistema político formal, el poder social está aún constituido sobre la base de criterios originados en la relación colonial (Quijano, 2014).

Aunado a esto, Breny Mendoza (2010) explica que la idea de raza surge con la idea del descubrimiento, utilizada para reclasificar socialmente y en forma estratificada a las personas en las colonias, según su cristianismo, la idea de pureza de sangre y las lenguas europeas. Así, la idea de raza reordena los regímenes de género preexistentes en las sociedades colonizadas. Mendoza (2010) también afirma que el eurocentrismo no solo conduce a:

la construcción de subjetividades e intersubjetividades entre europeos y no europeos que se basan en oposiciones binarias tales como civilización y barbarie, esclavos y asalariados, premodernos y modernos, desarrollados y subdesarrollados etc., sino que se toma por sentado la universalización de la posición epistémica de los europeos (p.22).

Sin embargo, María Lugones, filosofa feminista y educadora popular que centra su trabajo en lo que llama "opresiones múltiples" como lo son los términos, raza/ género/clase/sexualidad, concibe a la intersección de raza y género en términos estructurales amplios y asevera: "Quijano entiende que el poder está estructurado en relaciones de dominación, explotación, conflicto entre autores sociales que se disputan el control de los cuatro ámbitos básicos de la existencia humana: sexo, trabajo, autoridad colectiva, subjetividad/intersubjetividad, sus recursos y sus productos" (Lugones, 2008, p. 74).

Por lo que entender los rasgos históricamente específicos de la organización de género en el sistema moderno/colonial es determinante para una com- 
prensión de la ordenación diferencial del género en términos raciales, es así que, colonialidad no se refiere solo a la clasificación racial, sino que también es un fenómeno abarcador, ya que trata mediante el poder permear todo el control del acceso sexual, la autoridad colectiva y el conocimiento desde el interior mismo de las relaciones intersubjetivas (Lugones, 2008).

De tal manera que de una forma mitológica se entendió que Europa, como centro capitalista mundial que colonizó al resto del mundo, ya existía como centro de poder. Es entonces que, de acuerdo con una concepción de humanidad que se consolidó con esa mitología, la población mundial se diferenció en dos grupos: superior e inferior, racional e irracional, primitivo y civilizado, tradicional y moderno (Lugones, 2008). Por lo tanto, la interseccionalidad revela lo que se invisibiliza cuando las categorías raza y género se estudian por separado.

Lugones (2008) sostiene que las categorías invisibilizan a quienes somos dominadas y victimizadas bajo el concepto mujer y bajo las categorías raciales black, hispanic, asian, native american o chicana; es decir, a las mujeres de color, como ya he indicado, la autodenominación como tal no es equivalente a sí, sino que se propone en gran tensión con los términos que el Estado racista nos impone (p.82).

En el mismo sentido, Mendoza (2010) sustenta que Lugones se apoya en el trabajo de dos feministas, Oyuronke Oyewumi de origen nigeriana y Paula Allen Gunn, indígena de Estados Unidos, para comprobar cómo el género junto con la idea de raza fueron simultáneamente constructos coloniales para racializar y generizar las sociedades que sometían. Según estas feministas africanas e indígenas, no existían en las sociedades yorubas ni en los pueblos indígenas de América del Norte un principio organizador parecido al de género en occidente antes de la colonización. Sus culturas de origen no dividían ni jerarquizaban sus sociedades en base a género, teniendo las mujeres acceso igualitario al poder público y simbólico. Por lo que para estas feministas poscoloniales se reconocían más de dos géneros, lo cual debatía el dimorfismo sexual en occidente, el cual sirvió para encubrir, según Lugones, la forma en que las mujeres del tercer mundo experimentaron la colonización y continúan sobreviviendo

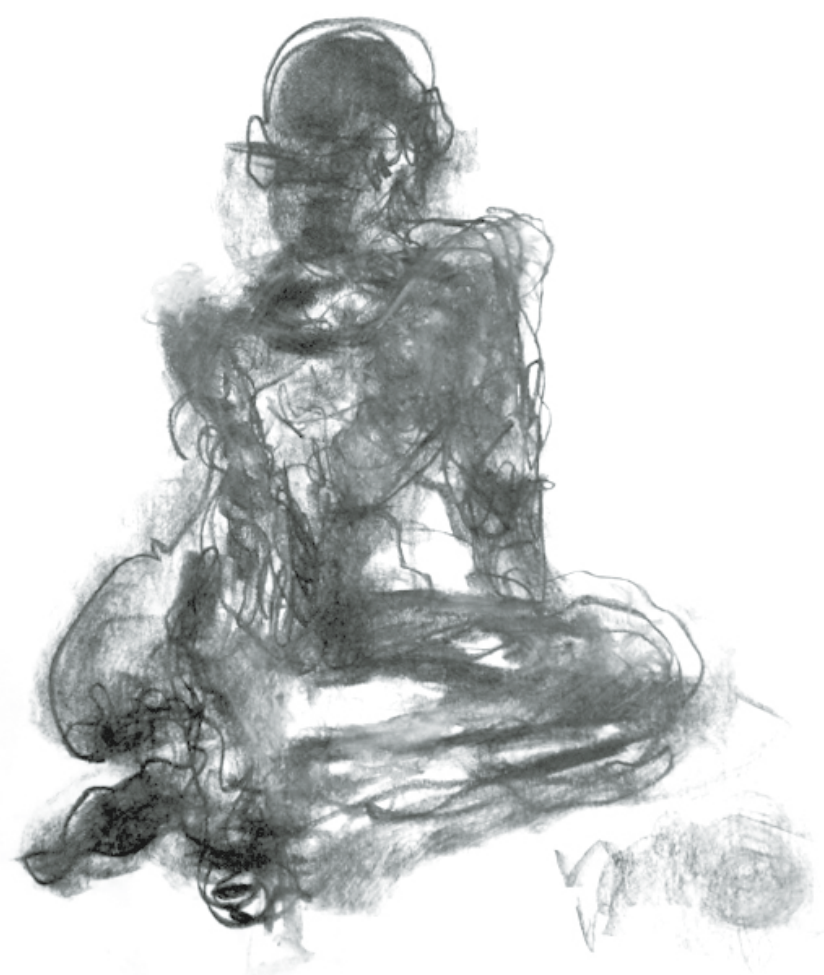
los efectos en la época poscolonial. 


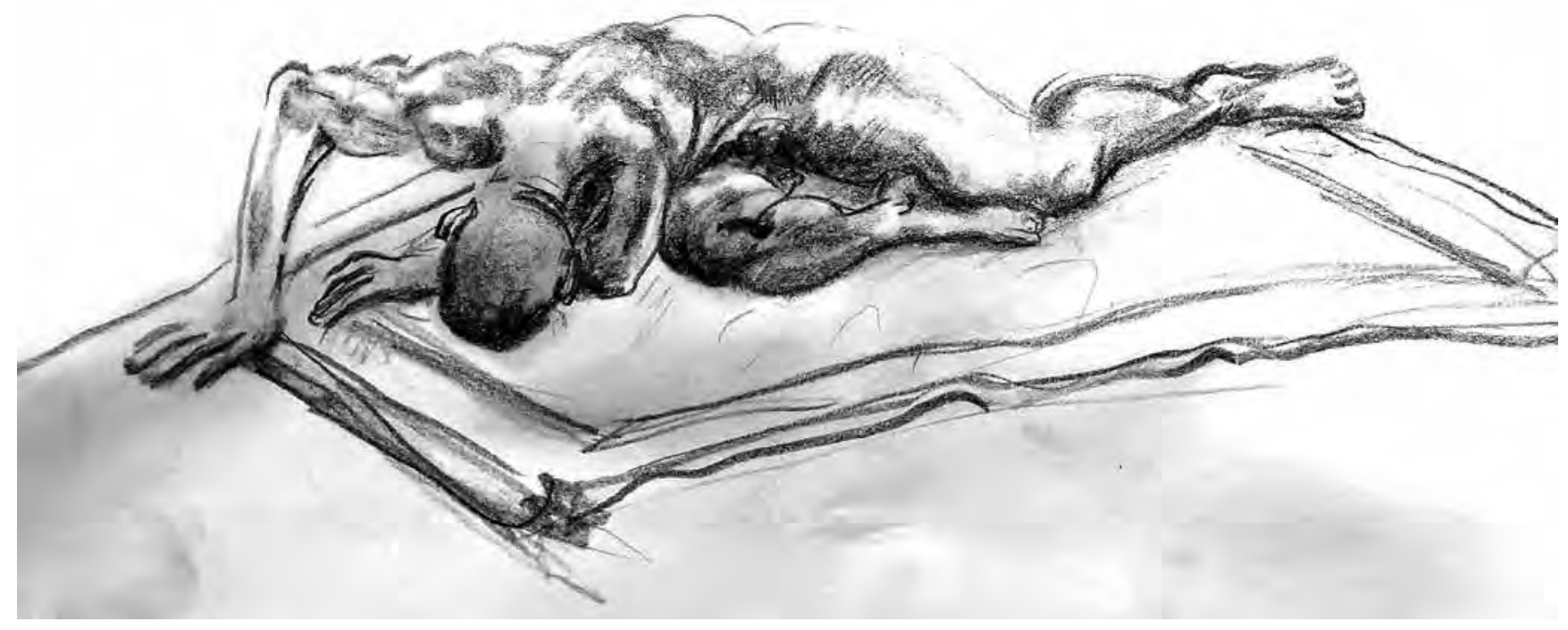

Por lo anterior, ignorar la historicidad y la colonialidad del género también evita que las mujeres blancas de occidente reconozcan la interseccionalidad de raza y género, así como su propia complicidad en los procesos de colonización y dominación capitalista y es por esta razón que a las feministas occidentales les parece difícil construir en la actualidad alianzas sólidas con las mujeres en sus países del tercer mundo.

Con esta mirada de la colonialidad del poder se evidencia que la interseccionalidad desenmascara la forma en que la mujer fue sometida a un proceso de blanqueamiento, el cual impuso la inseparabilidad de los términos mujer-blanca. Lugones (2008) plantea que: "mujer-blanca es una indivisible creación social que surge de la fusión entre occidente, colonia, raza, capital, heteronormatividad como componente constitutiva del sistema implantado en el siglo XVI con la conquista en nuestra América. Mujer es un efecto de la racialización del género" (citada en Alvarado, 2016, p.15).

Es así que las experiencias de educación superior con pueblos indígenas y afrodescendientes que se han desarrollado en América Latina en las últimas décadas brindan valiosas oportunidades de aprendizaje, por lo que, para comprender las relaciones actuales entre universidades y pueblos indígenas y afrodescendientes en
América Latina es necesario recordar algunos factores históricos, que si bien es cierto muestran mejoras, también es cierto que continúan afectando estas relaciones.

Daniel Mato (2012) sostiene que:

La historia de América ha sido marcada tanto por la conquista como también por la colonización europea, en la cual se evidencian masacres, despojos de territorios, desplazamientos y reorganización social de los pobladores originarios, sus religiones fueron prohibidas y obligadas a adoptar el catolicismo, igualmente, sus lenguas maternas fueron prohibidas en espacios públicos, especialmente en la escuela, cuando accedieron a ella (p.18).

En ese sentido, cuando se trata el recorrido histórico sobre pueblos indígenas y afrodescendientes en América Latina, en Argentina por ejemplo, el Estado desde su conformación mantuvo una política de genocidio e invisibilización hacia los pueblos indígenas y afrodescendientes a través del sistema educativo y otros medios como la ideología, el orden y la superioridad del hombre blanco, lo que naturalizó el exterminio sistemático de estos pueblos (Guaymás, 2018). 
Por lo tanto, la creación de universidades interculturales aparece como una construcción que parte del reconocimiento de la necesidad de tener una oferta educativa distinta, la cual transformará los espacios formativos de educación superior, por lo que comienzan a perfilarse como los espacios de mayor acogida de jóvenes indígenas en la educación superior, aunque existe también otro tipo de oferta educativa como la formación pedagógica (Olivera y Dietz, 2017).

Hoy en día existen en Argentina programas que incluyen políticas educativas en el nivel superior, entre ellos están los que proporcionan becas con cupos especiales creados en el año 2006; gracias a la administración del Instituto Nacional de Formación Docente (INFD) existe un programa de estímulos económicos para carreras de formación docentes pertenecientes a pueblos originarios, los cuales atienden a indígenas que realizan su formación profesional en las instituciones de educación superior (Guaymás, 2018).

En el caso del Estado Pluricultural de Bolivia, se presenta diversidad étnica y lingüística en los nueve departamentos que lo conforman, el idioma nacional es el castellano y también se hablan otros idiomas de las naciones y pueblos indígenas originarios. Aquí inició en el año 2005 el programa de fortalecimiento de liderazgo indígena a solicitud y en coordinación con los consejos educativos de pueblos originarios de Bolivia. Otra experiencia significativa la representa la Universidad Pública de El Alto (UPEA), fundada el 5 de septiembre del año 2000 bajo la ley 2115. Su creación se debió a las movilizaciones sociales en las que solicitaban una universidad pública y autónoma, es de esa forma que en el año 2003 se pone en vigencia esta ley para garantizar la autonomía universitaria de tal institución (Choque, 2013).

En tal sentido, Patricia Mena (2011) explica que el tema de la política intercultural cobra gran relevancia con la intervención de los organismos internacionales, de esta forma se podrá entender su inserción en los escenarios de los Estados y su inclusión dentro de las luchas de los movimientos étnicos en Latinoamérica. Esto se considera una fortaleza de los movimientos indígenas, la visibilidad que han tenido sus demandas a nivel internacional han permitido considerar a fondo la discusión en la agenda de los derechos humanos.
De esta manera, y para evitar situaciones de exclusión en el ámbito educativo, el sistema de Naciones Unidas ha promovido diferentes convenciones y declaraciones con el fin de proteger los derechos de las personas que, por su condición de raza, sexo y lugar de origen, han estado en situación de vulnerabilidad como colectivo minoritario o con menor poder dentro de la sociedad (Blanco, 2008).

Para el caso, en el ámbito de la educación, el instrumento de educación más importante es la Convención contra la Discriminación en Educación (UNESCO); sin embargo, no se pueden desligar otras convenciones como la Convención sobre la eliminación de todas formas de discriminación contra la mujer (ONU, 1979) o la Convención sobre la eliminación de toda forma de discriminación racial (1969) y la Declaración sobre los derechos de las personas pertenecientes a minorías nacionales o étnicas, religiosas y lingüísticas (1992) (Blanco, 2008).

\section{Es así que las experiencias de educación superior con pueblos indigenas $y$ afrodescendientes que se han desarrollado en América Latina en las últimas décadas brindan valiosas oportunidades de aprendizaje.}

Es importante preguntarse si las políticas educativas que se desarrollan contribuyen a la reducción de las desigualdades y al aumento de la cohesión social ya que, aunque la mayoría de los países adoptan los principios de la Declaración de educación para todos, en la práctica no se aplican.

Rosa Blanco (2008) sostiene que son distintos factores los que excluyen a numerosos alumnos del sistema educativo, "los niños y niñas llegan a la escuela en condiciones muy diferentes según el nivel de ingresos y el capital cultural de sus familias, sus posibilidades de acceso a las tecnologías de la información y la comunicación, o las características o recursos de las comunidades en que viven, entre otros" (Blanco, 2008, p. 3). 


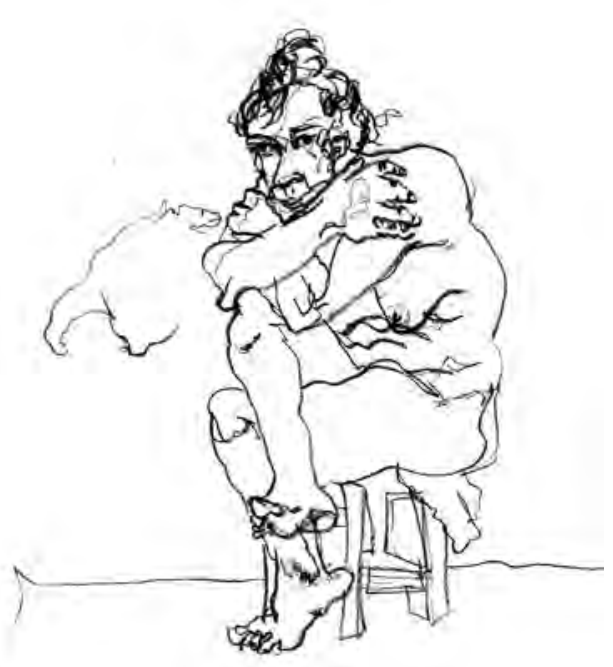

Estas desigualdades se reproducen en el ámbito escolar por factores como escuelas y universidades muy alejadadas e incompletas, las cuales limitan el acceso a la educación y la continuidad de estudios, de esa forma se sostiene la segmentación de centros educativos que tienen mayores necesidades, con docentes menos cualificados y menores recursos y procesos educativos que tienden a discriminar a aquellos estudiantes con un capital cultural distinto al dominante en la escuela. Es así como los sistemas educativos atrasan o expulsan a lo largo de la educación primaria a 4 de cada diez niños que ingresan oportunamente a primer grado (Blanco, 2008).

Es posible concluir que la exclusión en educación afecta no solo a aquellos que nunca han accedido a la escuela o son expulsados temporalmente de ella, sino también a quienes estando escolarizados sufren discriminación o son segregados por su origen social y cultural, por su género o sus niveles de rendimiento, y a quienes no logran aprender con calidad. La exclusión es, por tanto, un fenómeno de gran magnitud que amerita una revisión profunda de los factores que la generan, sean internos o externos a los sistemas educativos, así como es necesario el desarrollo de políticas intersectoriales que lo aborden de forma integral (Blanco, 2008).

En cuanto a las escasas estadísticas específicas sobre el acceso y graduación de individuos indígenas y afrodescendientes en el nivel superior, se obtienen otras estadísticas que pueden resultar útiles como indicadores indirectos de la existencia de situaciones en desventaja con respecto al acceso y egreso exitoso. En ese sentido, en el caso de Guatemala un estudio realizado en 1985 (Fabian y Urrutia, 2004) ofrecía una estimación en la que los estudiantes indígenas representaban solo el $6.2 \%$ del total de estudiantes matriculados en educación superior, proporción que contrasta notablemente con la cifra que dice que aproximadamente el $40 \%$ de la población de ese país es indígena (Mato, 2012, p.31).

En el caso de Brasil, se evidencia que el porcentaje del total de la población indígena del país que accede a la educación superior es de $0.6 \%$, magnitud que difiere del porcentaje de más del $10 \%$ para la población blanca y del $2 \%$ para la población negra y descendiente. En Colombia se señala que solo el $0,6 \%$ de los estudiantes matriculados en todas las modalidades de educación superior de ese país (técnica profesional, tecnológica universitaria) es indígena, lo cual refleja desigualdad ya que la proporción de población indígena en el total nacional es de aproximadamente 3,5\% (Mato, 2012, p.31). En México, se señalan datos referentes al año 2000 que muestran que sólo el 3,5\% de la población indígena de ese país es profesional, mientras que esta proporción es de aproximadamente $10,5 \%$ en el caso de la población total (Mato, 2012, p.31).

Complementariamente, puede tomarse en cuenta que según un estudio publicado en 2003 por la Asociación Nacional de Universidades e Instituciones de Educación Superior (ANUIES) en México, el 1\% de los jóvenes indígenas de entre 18 y 25 años ingresan a instituciones de educación superior y, de ellos, uno de cada cinco egresa y se titula, en contraste con el $22,55 \%$ de los jóvenes no indígenas de esa misma edad del país que acceden a dicha educación, egresan y se titulan (Mato, 2012, p.31).

En Ecuador, en el 2004 se estimaba que solo el 3\% de los jóvenes de ambos sexos de entre 18 y 24 años autoidentificados como indígenas asistían a instituciones de educación superior. Esta proporción era del $6 \%$ para el caso de quienes se reconocían como negros y del $8 \%$ para quienes se asumían como mulatos. Las tasas antes mencionadas contrastan con la del $14 \%$ correspondiente al grupo que se autoidentificaba como 
mestizo y la del 19\% para el que se proclamaba como blanco; así como con la tasa promedio nacional, que para entonces se calculaba en 14\% (Mato, 2012, p.32).

Existen limitantes de las poblaciones originarias al sistema superior de educación en cuanto al acceso, una de ellas es que los territorios indígenas presentan dificultades de conectividad e infraestructura de respaldo para el uso de las tecnologías de información y comunicación; además, muchas veces no existe estrategias de formación a docentes que reconozcan el enfoque diferencial étnico.

Es así como, Mazabel (2012) expone que:

en Colombia las Instituciones de Educación Superior en territorios con alta presencia de población afrocolombiana son débiles en la promoción de metodologías de enseñanza y contenidos acordes con las particularidades culturales de afrodescendencia. Además, los trabajos de grado y prácticas académicas de estudiantes afrocolombianos no están orientadas al desarrollo integral de sus territorios de origen (pp. 269-270).

Otro aspecto para tomar en cuenta es el contenido curricular de nivel superior, el cual debe estar integrado de tal forma que se incluya un currículo intercultural que tenga como objetivo promover la formación de profesionales comprometidos con el desarrollo económico, social y cultural, revalorando los saberes de los pueblos originarios con el fin de propiciar un proceso de síntesis con los avances del conocimiento científico para fomentar la difusión de los valores propios de las comunidades.

También se necesita que estudiantes de poblaciones originarias participen en el diseño y rediseño curricular para colocar sus conocimientos y las estrategias de convivencia entre saberes para que el aprendizaje colaborativo y significativo constituya uno de los pilares teóricos de una aproximación intercultural. Por otro lado, el modelo educativo intercultural también debe fomentar en la enseñanza basada en competencias y en los aprendizajes por lo que requiere de la investigación en todos los escenarios formativos que deben considerar el uso de las tecnologías, ser incluyente y privilegiar el respeto a las diferencias (Vera, 2007).

\section{Conclusiones}

Para lograr una equidad de género en la educación intercultural del nivel superior hay que sostener una postura crítica y analítica de todo lo concerniente a lo que significa atender a las poblaciones más desfavorecidas. En este caso se puede observar que los pueblos originarios y afrodescendientes tienen dificultad en el acceso, permanencia y egreso del nivel superior por razones de raza, género y clase, ya que cuando egresan del nivel básico o medio no logran, en su mayoría, ingresar al nivel superior debido a situaciones de violencia de género como son la falta de apoyo económico para continuar sus estudios, falta de oportunidades educativas, de becas, menor asistencia para ingresar y permanecer dentro del sistema sin necesidad de abandonar su lengua materna, menor grado de pertinencia, manejo inadecuado de las nuevas tecnología de información y comunicación, entre otras.

De la misma forma, se debe incorporar el enfoque de género en los espacios educativos del nivel superior para incluir a las mujeres indígenas y afrodescendientes en la participación de éstos, exigiendo el derecho que les corresponde como ciudadanas y asegurar que exista equidad en ambientes propicios a la discriminación y exclusión, ya que por ser mujer y pertenecer a un determinado pueblo originario se corre el riesgo de duplicar la violencia género.

Asimismo, se debe tomar en cuenta el estudio de la colonialidad del poder para analizar y comprender la interseccionalidad de raza, género y clase que explica el por qué históricamente se han dado situaciones de violencia simbólica hacia las mujeres indígenas y afrodescendientes en las que la mujer blanca tiene más oportunidad de gozar del derecho a la educación, sobre todo en la permanencia y en el egreso.

Aunado a lo previo, se deben respetar los convenios internacionales que protegen tanto el derecho a la educación, como también los derechos humanos de las mujeres, así como dar continuidad a todos los tratados internacionales de los que los Estados forman parte para proteger contra toda forma de discriminación, exclusión y violencia de género hacia las poblaciones originarias y afrodescendientes. 

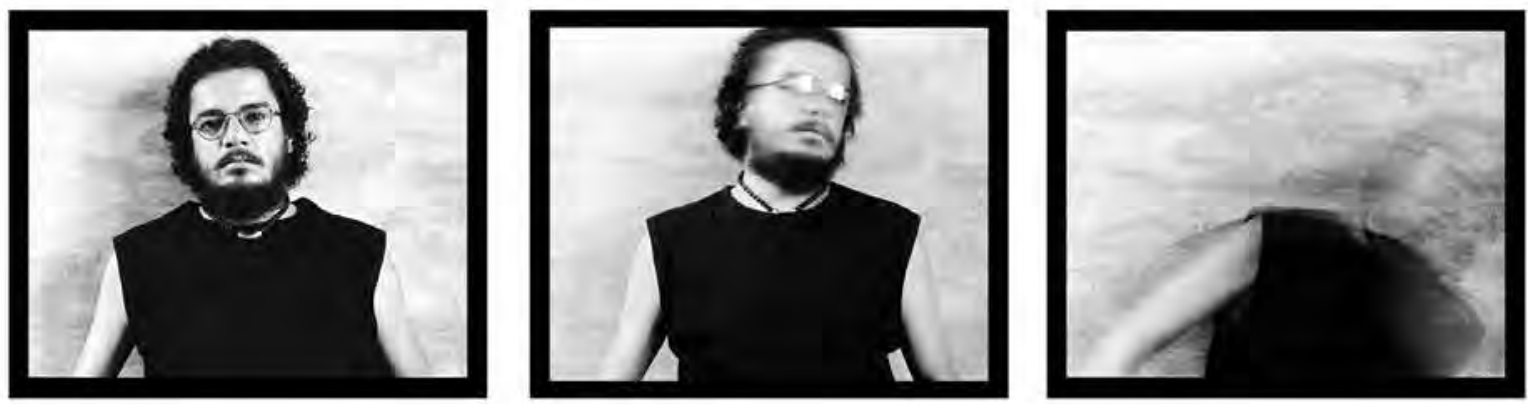

\section{En general nuestro organismo nos mantiene ajenos acerca de su trabajo.}

\section{Bibliografía}

Alvarado, M. (2016) Epistemologías feministas latinoamericanas: un cruce en el camino junto-a-otras, pero no-juntaa-todas. En: Religación. Revista de Ciencias Sociales y Humanidades desde América Latina, Vol. 1 no. 3. Quito, Ecuador.

Blanco, R. (2008) Haciendo efectivo el derecho a una educación de calidad sin exclusiones. Revista Colombiana de Educación, N. ${ }^{\circ} 54$. Primer semestre de 2008, Bogotá, Colombia.

Comisión Interamericana de Mujeres (2002) Programa Interamericano sobre la Promoción de los Derechos Humanos de la Mujer y la Equidad e Igualdad de Género. Disponible en https://www.oas.org/es/CIM/ docs/PIA[SP].pdf. Última visita 26 de agosto de 2019.

Choque, M. (2013) Educación Superior y pueblos indígenas y afrobolivianos retos y desafíos. Daniel M. (coord) Educación superior, diversidad e interculturalidad en América Latina. Caracas: UNESCOIESALC.

Duarte, J. García, J. (2016) Igualdad, Equidad de Género y Feminismo, una mirada histórica a la conquista de los derechos de las mujeres. Revista CS, núm. 18, Universidad ICESI.

Guaymás, A. (2013) Educación Superior y pueblos indígenas y afrodescendientes, en Argentina: logros, desafíos y recomendaciones. Daniel M. En (coord) Educación superior, diversidad e interculturalidad en América Latina. Caracas, UNESCO-IESALC.

Quijano, A. (2014) Colonialidad del poder y clasificación social. En Cuestiones y horizontes: de la dependencia histórico-estructural a la colonialidad/descolonialidad del poder. En Castro- Gómez, S. y Grosfoguel, R. (comps.) El giro decolonial: reflexiones para una diversidad epistémica más allá del capitalismo global. Buenos Aires: CLACSO.
Mato, D. (Coord.) (2013) Educación Superior y Pueblos Indígenas y Afrodescendientes en América Latina. Normas, Políticas y Prácticas en Mato, D. Educación superior, diversidad e interculturalidad en América Latina. Caracas: IESALC-UNESCO.

Mena, P. (2011) Las políticas interculturales en Educación Superior: continuidad o innovación en la formación de cuadros indígenas. Tesis para optar el grado de Doctora en el programa sociedades multiculturales y estudios interculturales. Universidad de Granada, España.

Mazabel, M. (2012) Decisiones, omisiones y contradicciones, Interculturalidad y Políticas Públicas en Educación Superior en Ecuador. En Mato, D.(coord) Educación superior, diversidad e interculturalidad en América Latina. Caracas: UNESCO-IESALC.

Mendoza, B. 2010. La epistemología del sur, la colonialidad del género y el feminismo latinoamericano, en Espinosa Y. (coord). Aproximaciones críticas a las prácticas teórico-políticas del feminismo latinoamericano, volumen L. Buenos Aires: En la frontera.

Lugones, M. (2008) Colonialidad y género. Tabula Rasa, núm. 9, juliodiciembre, 2008, Universidad Colegio Mayor de Cundinamarca Bogotá, Colombia, pp. 73-101.

Olivera, I., Dietz, G. (2017) Educación superior y pueblos indígenas: marcos nacionales para contextualizar. Antropológica del Departamento de Ciencias Sociales, vol. XXXV, núm. 39, 2017 Pontificia Universidad Católica del Perú.

Vera, J. (2007) Teoría y Método en el Diseño Curricular Intercultural por Competencias. Ra Ximhai Vol. 3. Número 2, Mayo - agosto 2007, pp. 397-416. 